\title{
Correction to: Metaheuristics for Combinatorial Optimization
}

Salvatore Greco, Mario F. Pavone, El-Ghazali Talbi, and Daniele Vigo

\section{Correction to:}

S. Greco et al. (Eds.): Metaheuristics for Combinatorial

Optimization, AISC 1332,

https://doi.org/10.1007/978-3-030-68520-1

The book was inadvertently published with the incorrect volume number (1336) and this has been corrected now (1332). 